\title{
QUANTITATIVE THEORY OF EFFECTIVENESS OF HIGHEST EDUCATION: ROLE OF INTERPERSONAL COMMUNICATIONS
}

\author{
Ibragim Suleimenov, Dr.Sci, PhD \\ Dina Shaltykova, PhD \\ Polina Obukhova, MS \\ Anastasiya Stentsova
}

Almaty University of Power Engineering and Telecommunications, Almaty,

Kazakhstan

Kamilya Suleymenova, Dr. Sci

Birmingham University, UK

\begin{abstract}
The influence of interpersonal communications inside and outside a University on effectiveness of education is investigated. It is shown, that low effectiveness of direct (traditional) education may be compensated by increasing of communicational activeness of students and stimulation of interpersonal communications. Hysteresis dependence of quality of education on communication activeness of students takes place at low effectiveness of direct education. The fact allows explaining of modern situation in post-soviet countries, particularly, in Kazakhstan, when sufficient efforts of the government gives no evident results in respect to increasing of education quality.
\end{abstract}

Keywords: Interpersonal communications, post-industrial society, quality of education, hysteresis

\section{Introduction}

Improving the quality of higher education is important for the vast majority of countries around the world. The transition from industrial to post-industrial society is a challenge for modern pedagogic sciences (Pereslegin S.,2011.): higher education built on the industrial paradigm is far from being fully able to meet the demands of the post-industrial labor market (Pereslegin S.,2011.) As noted in (Suleimenov, I. E. et. al., 2011), the postindustrial labor market is characterized by relatively rapid emergence of new professions (or a substantial transformation of existing ones), and many of 
them require diverse skills. Modern High School, operating in the industrial paradigm, just do not have time to form the corresponding specialty. As a result, the training for many professions, in particular, the ones that appear in real time is actually performed outside of university programs.

As a consequence, in modern conditions the significance of selfeducation increases, specifically the education that is formed by the informal institutions or formal institutions not related to universities.

Consequently, the quantitative description of the factor «educational media» is relevant for the theory of education; it promotes learning of the individual in an informal way.

The quantitative model, which allows taking into account both the impact of educational media and direct training, is proposed in present article. This model allows identifying the conditions when the educational media is crucial. It also allows us to show that there are conditions under which the indicators of the quality of education change abruptly at gradual modification of the control parameters. This serves as a basis for the application of the "strategy of a miracle" (Pereslegin S., 2011.) to higher education in countries such as Kazakhstan, where post-transitional crisis phenomena (Suleymenova K., 2012), that affect higher education are apparent (Suleimenova K.I., 2013).

\section{Basic Model}

A quantitative description of the effectiveness of educational programs, as will be seen below, can be developed on the basis of analogies with models that describe the promotion of innovations; the most known example is Bass's diffusion model (BDM). Thereare a number of results(Bass F. M.,1997) showing that this modeladequately describes thedynamics ofpromotion of innovations.

One can say that promotion of innovation is determined by twotypes ofinformational impacton the consumer.One ofthem is determined bythe influence of advertising and mass-media, and the other - by the transmission of informationdirectlyfrom one consumer to another («Word-in-mouth»). Accordingly, the Bass's equation using in the classical formconsists of two termsreflectingthe mentioned informational impacts.

$$
\frac{d N}{d t}=\alpha\left(N_{0}-N\right) N+\beta\left(N_{0}-N\right)
$$

where coefficients $\alpha$ and $\beta$ characterize the intensity of informational influences that determine the dynamics of the marketing of an innovation, $N_{0}$ - market potential for this product or service, $N(t)$ - the number of 
consumers at a moment $t$. Traditionally, the term proportional to $\alpha$ is associated with interpersonal communication, the term proportional to $\beta$ with the impact of the mass media and advertisement.

The analogy between the BDM and the proposed description of the effectiveness of educational programs and promotion of innovations is the following. As in the case of promotion of innovations, the volume of knowledge assimilated by a student is determined by two factors; one of them is the direct informational impact by a teacher during lessons, and the other is the result of interpersonal communications. For example, as shown by the results of the survey conducted in several Technical Universities in Kazakhstan, most of the skills in software application the inquired students received in interpersonal communications; the mutual influence of the students in the course of training further will be denoted by the term WordIn-Mouth Education (WIME). This term additionally underlines the analogy between the approach being developed and BDM and its modifications.

Formalization of education process may be developed on the base of theory on information transmission as follows. "Professional knowledge" may be considered as a set of information packages that should be assimilated by a student for obtaining correspondent qualification. Consequently the diagram, Fig. 1 is applicable: a student obtains next information package and his knowledge is growing from level to level. It is supposed that transition on the highest level $K$ corresponds to obtaining of complete professional knowledge.

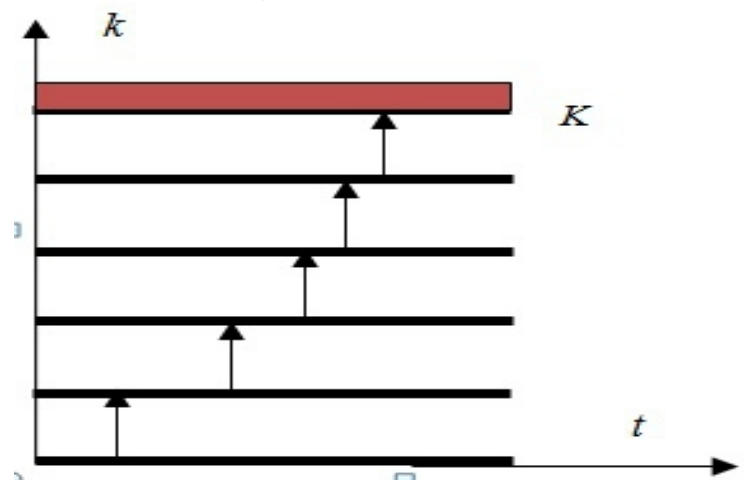

Fig.1. Formal scheme of learning paths during education period.

Statistically, transitions between the levels showed on Fig.1 may be described by next system of differential equations.

$$
\frac{d N_{0}}{d t}=-N_{0} \sum_{j=1} \alpha_{j 0} N_{j}-\beta_{0} N_{0}+\frac{1}{\tau_{1}} N_{1}
$$




$$
\begin{aligned}
& \frac{d N_{k}}{d t}=-\sum_{j=1} \alpha_{j k} N_{j} N_{k}+\sum_{j=1} \alpha_{j k-1} N_{j} N_{k-1}-\beta_{k} N_{k}+\beta_{k-1} N_{k-1}+\frac{N_{k+1}}{\tau_{k+1}}-\frac{N_{k}}{\tau_{k}} \\
& \frac{d N_{K}}{d t}=\sum_{j=1} \alpha_{j, K-1} N_{j} N_{K-1}+\beta_{K-1} N_{K-1}-\frac{1}{\tau_{K}} N_{K},
\end{aligned}
$$

where $N_{k}$ is a number of students corresponding to level of knowledge $k$, coefficients $\alpha_{j k}$ reflects information impact of interpersonal communications resulting in growing of knowledge, coefficients $\beta_{j}$ - direct information impact during lessons, etc. It is taking into account that a student may forget some information i.e. there are not only direct transitions between the levels showing on Fig.1, but inverse ones too. Inverse transitions are described by terms $N_{j} / \tau_{j}$. These terms take into account restricted time of education too; a student may leave university after finishing of education period and even earlier.

The system (2) - (4) is quite complicated for direct analyses; nevertheless some important results may be obtained with the help of simplified model that includes 3 levels only. Corresponding system of differential equations may be written as follows.

$$
\begin{aligned}
& \frac{d N_{0}}{d t}=-N_{0}\left(\alpha_{20} N_{2}+\alpha_{10} N_{1}\right)-\beta_{0} N_{0}+\frac{1}{\tau_{1}} N_{1} \\
& \frac{d N_{1}}{d t}=-N_{1}\left(\alpha_{21} N_{2}+\alpha_{11} N_{1}\right)+N_{0}\left(\alpha_{20} N_{0}+\alpha_{10} N_{0}\right)+\beta_{0} N_{0}-\beta_{1} N_{1}-\frac{1}{\tau_{1}} N_{1}+\frac{1}{\tau_{2}} N_{2} \\
& \frac{d N_{2}}{d t}=N_{1}\left(\alpha_{21} N_{2}+\alpha_{11} N_{1}\right)+\beta_{1} N_{1}-\frac{1}{\tau_{2}} N_{2}
\end{aligned}
$$

Summing Eq. (5) - (7) one can obtain that the total sum of students having different levels of knowledge should be a constant.

$$
N_{0}+N_{1}+N_{2}=C
$$

I.e. the considering system is reduced to next two differential equations.

$$
\frac{d N_{0}}{d t}=-N_{0}\left(\alpha_{20} N_{2}+\alpha_{10}\left(C-N_{2}-N_{0}\right)\right)-\beta_{0} N_{0}+\frac{1}{\tau_{1}}\left(C-N_{2}-N_{0}\right)
$$


$\frac{d N_{2}}{d t}=q\left(C-N_{2}-N_{0}\right)\left(\alpha_{20} N_{2}+\alpha_{10} N_{1}\left(C-N_{2}-N_{0}\right)\right)+q \beta_{0}\left(C-N_{2}-N_{0}\right)-\frac{1}{\tau_{2}} N_{2}$

The next connection

$\left(\alpha_{20}, \alpha_{10}, \beta_{0}\right)=q\left(\alpha_{21}, \alpha_{11}, \beta_{1}\right)$

is used in Eqs. (8) - (10). Connection (11) is likely to be valid while the considering coefficient are determined by product of frequency of information impacts on their effectiveness.

Let us consider the equilibrium state, then

$$
\begin{aligned}
& N_{0}\left(\alpha_{20} N_{2}+\alpha_{10} N_{1}\right)+\beta_{0} N_{0}-\frac{1}{\tau_{1}} N_{1}=0 \\
& N_{1} q\left(\alpha_{20} N_{2}+\alpha_{10} N_{1}\right)+q_{1} \beta_{0} N_{1}-\frac{1}{\tau_{2}} N_{2}=0
\end{aligned}
$$

From Eqs. (12), (13) one can easy obtain

$$
\frac{N_{1}}{N_{0}}=\frac{\tau_{1}}{q \tau_{2}} \frac{N_{2}}{N_{1}}=k
$$

Multiplier $k$ may be used for further simplification of Eqs. (12), (13). Namely,

$$
N_{1}=k N_{0}, \quad N_{2}=\frac{q \tau_{2}}{\tau_{1}} k^{2} N_{0}=q_{1} k^{2} N_{0}
$$

where $q_{1}=\frac{\tau_{2}}{\tau_{1}} \frac{\beta_{0}}{\beta_{1}}$.

Substituting relations (15) in (12), (13) and (8) one can easily show that considering systems of equations may be reduced to the only algebraic equation of third order.

$$
q_{1} k^{3}-\left(\tau_{1} \beta_{0} q_{1}+\tau_{1} \alpha_{20} C q_{1}-1\right) k^{2}-\left(\tau_{1} \beta_{0}+\tau_{1} \alpha_{10} C-1\right) k-\tau_{1} \beta_{0}
$$


The relative number of students having complete qualification in this model is given as

$$
\frac{N_{2}}{C}=\frac{q_{1} k^{2}}{1+k+q_{1} k^{2}}
$$

\section{Results}

Some examples of numerical calculation of relative number of students having complete qualification (17) are presented on Fig.2,3.

It can be seen that for the both values used here $q_{1}=0,5$ и $q_{1}=1,5 \mathrm{a}$ similar behavior with decreasing the $\beta_{0} \tau_{1}$ parameter is observed, which reflects effectiveness of direct education (education during lessons, etc.). Namely, obtained dependences are close to straight lines at relatively large values of $\beta_{0} \tau_{1}$, but if parameter $\beta_{0} \tau_{1}$ is relatively low, then the coefficient of effectiveness of education $N_{2} / C$ changes by jump at certain value of $\alpha_{20} \tau_{1}$.

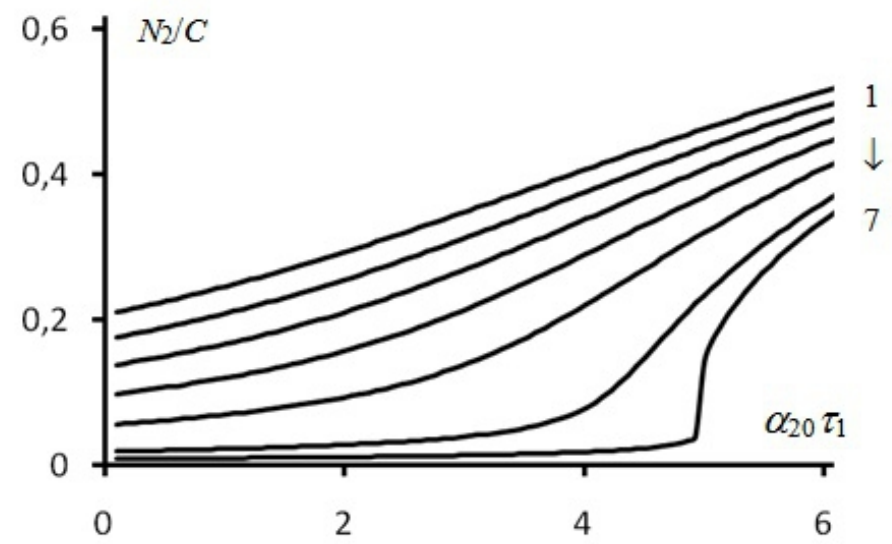

Fig.2. A set of dependences of relative number of students having complete qualification $N_{2} / C$ on parameter $\alpha_{20} \tau_{1}$ at different values of $\beta_{0} \tau_{1} ; q_{1}=0,5, \alpha_{10} \tau_{1}=0,1 ; \beta_{0} \tau_{1}=1,2(1), 1,0$ (2), 0,8 (3), 0,6 (4), 0,4 (5), 0,2 (6), 0,125 (7).

A further reduction of the parameter $\beta_{0} \tau_{1}$ leads to S-shaped relationships (Fig.4, 5) $N_{2} / C$ on $\alpha_{20} \tau_{1}$. 


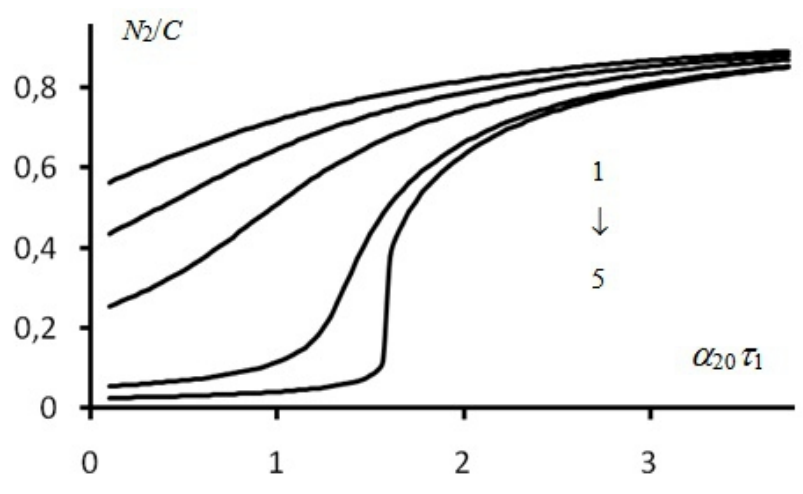

Fig.3. A set of dependences of relative number of students having complete qualification $N_{2} / C$ on parameter $\alpha_{20} \tau_{1}$ at different values of $\beta_{0} \tau_{1} ; q_{1}=1,5, \alpha_{10} \tau_{1}=0,5 ; \beta_{0} \tau_{1}=1,1(1), 0,8$ (2), 0,5 (3), 0,19 (4), 0,125 (5).

It should be emphasized that the construction of Fig.4, 5 used relatively large values of the parameter $q_{1}$, characterizes the ratio of the effectiveness of learning at higher and lower levels. A very low effectiveness of first level training index was also used, which corresponds to the reality in countries such as Kazakhstan. Nevertheless, as is evident from Fig. 4, 5, there are conditions when the low quality of "direct" education can be compensated by WIME, i.e. learning through interpersonal communications.

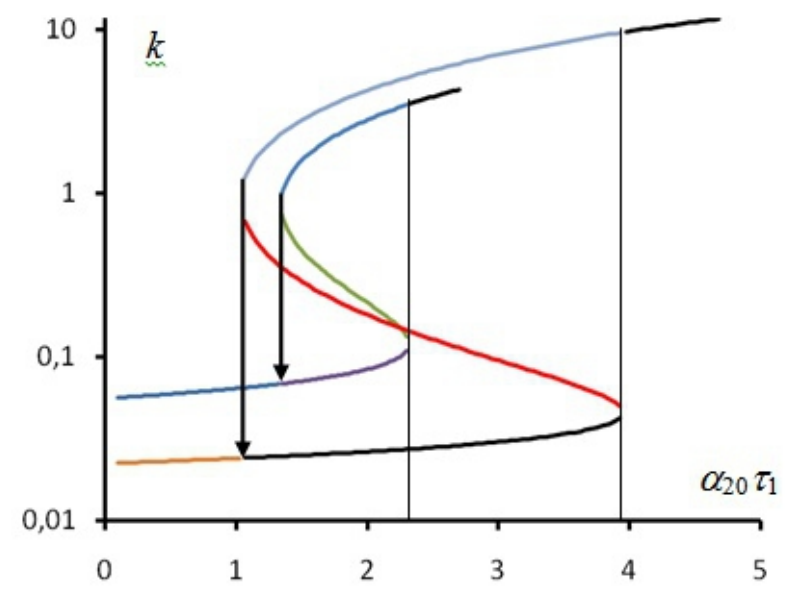

Fig.4. Dependences of coefficient $k$ on parameter $\alpha_{20} \tau_{1}$ at the next values of other parameters: $q_{1}=2,0, \alpha_{10} \tau_{1}=0,1 ; \beta_{0} \tau_{1}=0,05(1), q_{1}=2,7, \alpha_{10} \tau_{1}=0,1 ; \beta_{0} \tau_{1}=0,02(2)$ 


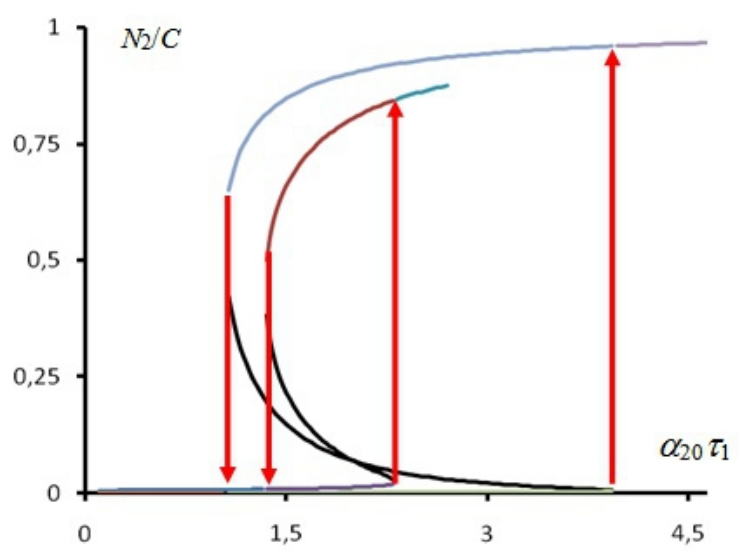

Fig.5. Dependences of relative number of students having complete qualification $N_{2} / C$ on parameter $\alpha_{20} \tau_{1}$ at the next values of other parameters: $q_{1}=2,0, \alpha_{10} \tau_{1}=0,1 ; \beta_{0} \tau_{1}=0,05$ (1), $q_{1}=2,7, \alpha_{10} \tau_{1}=0,1 ; \beta_{0} \tau_{1}=0,02$ (2)

\section{Discussion}

The product of the lifetime of information packet in the memory of a student who has reached the lowest level in the efficiency of acquisition of the package, calculated in terms of frequency $\beta_{0} \tau_{1}$, obviously, is a measure of the effectiveness of training in the primary stages. In the current environment, at least for the post-Soviet states, this value is known to be low, in fact, to enhance it by traditional (purely administrative) measures is not possible.

At the same time, the results show that improving the quality of education in general does not necessarily require increasing the quality of the "direct" training. For small values of correspondent parameter, its increase even by tens of percent will not give the expected result. Much more effective is the use of the factor of interpersonal communication, i.e. creating an environment in which information is shared among students. (As shown by the results of surveys, in this way most of the students get the skills in using the software, i.e. the factor considered already acts as a kind of informal institution).

Stimulating interpersonal communication makes possible qualitative change in the situation. As it follows from Figure 4, 5, with a gradual increase in indicators of the effectiveness of such communications, we can expect a quantum leap that from the mathematical point of view corresponds to the transition from one branch of solution to another. Moreover, the existence of multiple branches of solutions suggests that in this situation the "strategy of a Miracle", understanding in accordance with (Pereslegin S.,2011.), appears as possible for realization. This strategy corresponds to the transition of the system from one branch to another through the use of factors 
that are not included in the proposed model ("quantum tunneling"). The creation of innovative research and educational clusters can be considered one of these factors; the corresponding program is now formed in the National Engineering Academy of the Republic of Kazakhstan.

\section{Conclusions}

Thus, analogy with well-known theories of innovation promotion is of interest for building up of quantitative theories aimed to describe quality of education in frameworks of paradigm of theory of information.

Proposed theory gives possibility to show, that stimulation of interpersonal communications is of significance importance for increasing of quality of education, particularly. Moreover, this factor may leads to very high quality of education even in the situation, when effectiveness of traditional forms of education (direct education) is very low.

Proposed theory predicts the existence of few stable states in educational systems in quite wide region of conditions too. Some observations allow concluding that level of education in post-soviet countries corresponds to the lowest branch of solution of correspondent equations, i.e. education in these countries is at the lower possible position. The existence of other branches of solutions that meet a substantially higher quality of education implies that the formation efficiency can be increased by a jump through the use of unconventional measures, which together constitute the "strategy of a miracle" in the terminology of S.Pereslegin.

\section{References:}

Pereslegin S. Ockham's dangerous razor. Moscow. 2011.

Suleimenov, I. E., Mun, G. A., Grigoriev, P. E., Negim, E. S. M., Yeligbayeva, G. Z., \& Suleimenova, K. I. (2011). Higher Education and Science: Portrait Against the Background of Global Crisis. World Applied Sciences Journal, 15(9).

Suleymenova Kamilya, (2012), Institutions, efficacité économique et autoritarisme politique: cas de la Chine et de la Russie, Thèse de doctorat: Sciences économiques, Centre d'Etudes en Macroéconomie et Finance Internationale, Nice

Suleimenova K.I., Obukhova P.V., Shaltykova D.B., Suleimenov I.E. (2013) Post-transition period and quality of higher education: ways to overcome the crisis phenomena. International Letters of Social and Humanistic Sciences 8. Bass F. M., A new product growth model for consumer durables, Mgmt Sci., (1969).

Parker P. M., Aggregate diffusion forecasting models in marketing: a critical review, Int. J. Forecasting, (1994) 
Chatterjee, R. and J. Eliashberg. The Innovation Diffusion Process in a Heterogeneous Population: A Micromodeling Approach. Management Science, 36(9), (1990).

Skiadas C. H.,Giovanis A. N., A stochastic Bass innovation diffusion model for studying the growth of electricity consumption in Greece, Applied stochastic models and data analysis, (1997) 